\title{
Generation of $100+++$ Gbit/s signals using multilevel modulation formats
}

Tokle, Torger; Serbay, M.; Jensen, Jesper Bevensee; Rosenkranz, W.; Jeppesen, Palle

Published in:

LEOS Summer Topical Meetings, 2007 Digest of the IEEE

Link to article, DOI:

10.1109/LEOSST.2007.4288431

Publication date:

2007

Document Version

Publisher's PDF, also known as Version of record

Link back to DTU Orbit

Citation (APA):

Tokle, T., Serbay, M., Jensen, J. B., Rosenkranz, W., \& Jeppesen, P. (2007). Generation of 100+++ Gbit/s signals using multilevel modulation formats. In LEOS Summer Topical Meetings, 2007 Digest of the IEEE (pp. 256-257). IEEE. https://doi.org/10.1109/LEOSST.2007.4288431

\section{General rights}

Copyright and moral rights for the publications made accessible in the public portal are retained by the authors and/or other copyright owners and it is a condition of accessing publications that users recognise and abide by the legal requirements associated with these rights.

- Users may download and print one copy of any publication from the public portal for the purpose of private study or research.

- You may not further distribute the material or use it for any profit-making activity or commercial gain

- You may freely distribute the URL identifying the publication in the public portal 


\title{
Generation of $100+++$ Gbit/s signals using multilevel modulation formats
}

\author{
Torger Tokle, Murat Serbay, Jesper Bevensee Jensen, Werner Rosenkranz and Palle Jeppesen.
}

\begin{abstract}
Multilevel modulation formats are used as the enabling technology to obtain $120 \mathrm{Gbit} / \mathrm{s}$ per channel bit rates, and $240 \mathrm{Gbit} / \mathrm{s}$ when polarisation multiplexing is added. Using OTDM, this can be extended even further.
\end{abstract}

\section{INTRODUCTION}

The use of multilevel modulation formats is an effective way to increase the bit rate of optical communication systems. Many experiments in the recent years have demonstrated that multilevel modulation formats offer many benefits compared to binary modulation at the same bit rate. The benefits include the use of low-bandwidth electrical generation and reception equipment, enhanced tolerance towards chromatic dispersion and polarisation mode dispersion, and enhanced spectral efficiency in wavelength division multiplexing (WDM) systems.

By combining phase and amplitude modulation, even higher bit rates can be obtained. Already in 2003, Liu, et al. demonstrated the combination of differential binary phase shift keying (DBPSK) and amplitude shift keying (ASK) at 10 Gbaud [1]. Since then, a number of experiments at higher symbol rates have extended the maximum per channel bit rate. Furthermore, the bit rate per wavelength can be doubled by transmitting two modulated signals with orthogonal polarisations (PolMUX).

In this article, we present several experiments on multilevel modulation formats to obtain bit rates above what is obtainable by using binary transmitters. We present a demonstration of $240 \mathrm{Gbit} / \mathrm{s}$ per channel bit rate obtained by combining differential quadrature phase shift keying (DQPSK), ASK and PolMUX. It is clearly demonstrated that multilevel modulation is an efficient method to increase the bit rate of optical communication systems.

\section{Multilevel Modulation Formats}

Multi-level modulation formats allow realisation of transmission links at higher bit rates than available from binary formats. Even when using state of the art electronics the bit rate can be doubled or even tripled by using quaternary or 8-ary modulation formats operating at the same symbol rate.

Several experiments using DQPSK have demonstrated bit rates of $80 \mathrm{Gbit} / \mathrm{s}$ to $100 \mathrm{Gbit} / \mathrm{s}[2,3]$. We further applied an additional ASK modulation to the DQPSK in order to create an 8-ary modulation at a symbol rate of

T. Tokle, J. Bevensee Jensen and P. Jeppesen are with COM•DTU Department of Communications, Optics, and Materials, Technical University of Denmark, Ørsteds Plads, Building 345V, DK-2800 Kgs. Lyngby, Denmark (email:tt@com.dtu.dk)

M. Serbay and W. Rosenkranz are with Chair for Communications University of Kiel, Kaiserstraße 2, 24143 Kiel, Germany

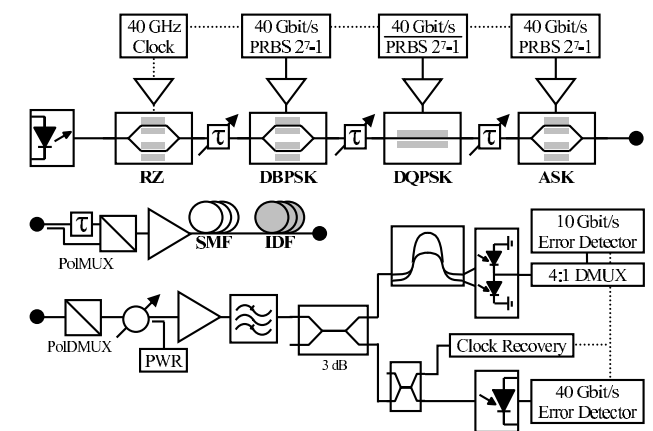

Fig. 1. Experimental setup for $240 \mathrm{Gbit} / \mathrm{s}$ RZ-DQPSK-ASK

40 Gbaud, and thus generate a $120 \mathrm{Gbit} / \mathrm{s}$ signal using only commercially available electronics.

As illustrated in Figure 1, the signal was generated by a concatenation of four modulators, one Mach-Zehnder modulator (MZM) for pulse carving, one MZM for ASK modulation, one MZM for DBPSK modulation and finally one phase modulator for DQPSK modulation [4]. With three bits per symbol, the resulting bit rate of the RZ-DQPSKASK signal was then $120 \mathrm{Gbit} / \mathrm{s}$. By further applying PolMUX, the bit rate was doubled to $240 \mathrm{Gbit} / \mathrm{s}$.

When combining amplitude modulation and phase modulation, the amplitude extinction ratio is a trade-off between good eye opening for the amplitude signal and good eye opening for the demodulated phase signal. We found that an extinction ratio of $4.5 \mathrm{~dB}$ resulted in optimum performance for the system as a whole, significantly lower than the theoretical value due to amplitude jitter on the driving signals [4].

In the pre-amplified receiver, the signal was split into two branches, one for detection of the phase modulation and one for ASK detection. The ASK signal was directly received by a $50 \mathrm{GHz}$ photodetector, while the phase information was demodulated using a one-symbol delay interferometer followed by a $45 \mathrm{GHz}$ balanced photodetector.

As seen in Figure 3(a) the width of the optical power spectrum is exactly the same for $120 \mathrm{Gbit} / \mathrm{s}$ RZ-DQPSKASK and for $40 \mathrm{Gbit} / \mathrm{s}$ DBPSK. Thus we can increase the bit rate by a factor of three (six with PolMUX) without increasing the spectral width.

The receiver sensitivity is illustrated in Figure 3(b). Compared to the sensitivity of the pure $80 \mathrm{Gbit} / \mathrm{s}$ RZDQPSK, the additional amplitude modulation caused a power penalty of $10 \mathrm{~dB}$, which is primarily caused by the low extinction ratio of the ASK signal, and the resulting distortions on the demodulated DQPSK eye.

Next we transmitted the signal over a $50 \mathrm{~km}$ fibre span, 

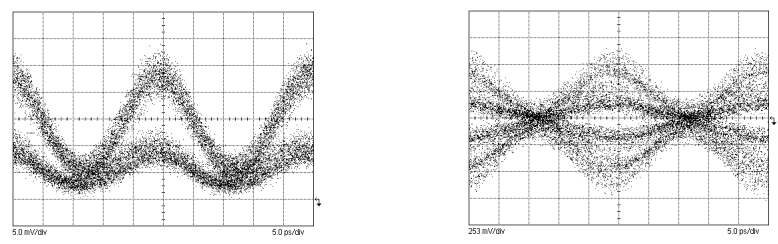

Fig. 2. 40 Gbaud ASK (left) and DQPSK (right) tributaries of the 120 Gbit/s RZ-DQPSK-ASK signal after polarisation DMUX.
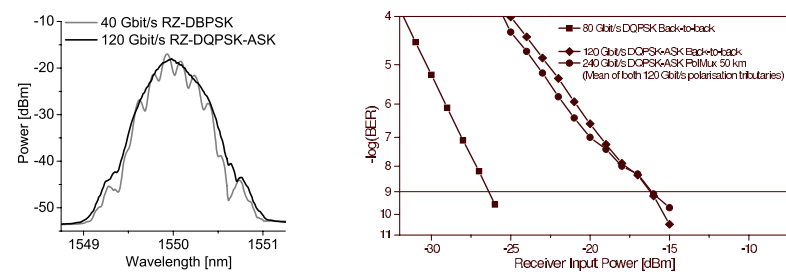

Fig. 3. Optical power spectra of binary 40 Gbit/s DBPSK and multilevel $120 \mathrm{Gbit} / \mathrm{s}$ RZ-DQPSK-ASK (left). BER vs. receiver input power for $80 \mathrm{Gbit} / \mathrm{s}$ DBPSK, $120 \mathrm{Gbit} / \mathrm{s}$ RZ-DQPSK-ASK and $240 \mathrm{Gbit} / \mathrm{s}$ RZ-DQPSK-ASK PolMUX (right)

and measured the bit error rate (BER) performance, as shown in Figure 3(b). There was no power penalty induced by the transmission, and no penalty from the polarisation multiplexing.

Polarisation multiplexing is a rather simple method to double the bit rate of an optical communication system. The two states of polarisations are used as independent channels, and the fibre bandwidth is thus doubled. However, it is necessary to track any changes to the state of polarisation of the incoming signal in order to maintain good polarisation demultiplexing. This can easily be implemented in the laboratory, but is more challenging in real systems. Furthermore, polarisation mode dispersion (PMD) will significatively degrade a polarisation multiplexed signal.

\section{OTDM and Multilevel Modulation}

One of the more promising approaches to obtain ultrahigh per channel bit rates is to combine optical time division multiplexing (OTDM) with multilevel modulation formats. We demonstrated this in an early experiment, where DBPSK was combined with ASK in order to transmit two bits for every OTDM symbol, thus doubling the bit rate [5].

DBPSK and ASK modulation, both at $10 \mathrm{Gbit} / \mathrm{s}$ was added to a $10 \mathrm{GHz}$ pulse train with a pulse width of $1.5 \mathrm{ps}$. This 20 Gbit/s RZ-DBPSK-ASK signal was then optically multiplexed to a $160 \mathrm{Gbit} / \mathrm{s}$ OTDM signal. In the receiver, the signal was demultiplexed back down to the base symbol rate of 10 Gbaud using a non-linear optical loop mirror, and the DBPSK and ASK tributaries were detected simultaneously. Eye diagrams of the received signal is shown in Figure 4. The optimum extinction ratio of the ASK tributary was found to be $7 \mathrm{~dB}$. Using multilevel modulation, we doubled the bit rate, at the cost of about $8 \mathrm{~dB}$ penalty
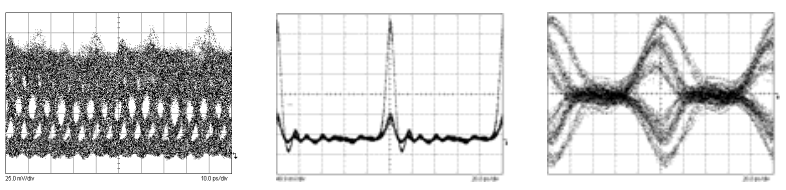

Fig. 4. 160 Gbit/s RZ-DBPSK-ASK OTDM signal (left), demultiplexed $10 \mathrm{Gbit} / \mathrm{s}$ ASK tributary (centre) and demodulated $10 \mathrm{Gbit} / \mathrm{s}$ DBPSK tributary (right).

compared to the binary system.

As always in DBPSK-ASK systems, both the ASK and DBPSK signal suffers from the compromised extinction ratio, but in this experiment the main limiting factor was the high timing jitter of 500 fs of the optical pulse train used to demultiplex the OTDM signal. This limited the maximum OTDM symbol rate to 80 Gbaud and thus the total bit rate to $160 \mathrm{Gbit} / \mathrm{s}$.

\section{Summary AND OUTLOOK}

We have utilised multilevel modulation formats to obtain per channel bit rates higher than what is achievable from binary transmitters. By using up to three bits per symbol, a maximum bit rate of $240 \mathrm{Gbit} / \mathrm{s}$ was achieved.

Looking forward, 4 bits per symbol has been experimentally demonstrated at symbol rates of 10 Gbaud [6,7]. Furthermore, it has been demonstrated that the combination of multilevel modulation formats and OTDM can be extended to several Tbit/s. In [8], OTDM, DQPSK and PolMUX were combined to generate a $2.56 \mathrm{Tbit} / \mathrm{s}$ signal with a 640 Gbaud symbol rate. It is clear that multilevel modulation formats are a key enabling technology for ultra-high per channel bit rates.

\section{ACKNOWLEDGMENT}

The Danish Council for Strategic Research funded the MultiSpeed project, and COST action 291 sponsored a short term scientific mission for M. Serbay.

\section{REFERENCES}

[1] X. Liu, et al., "Quaternary differential-phase amplitude-shiftkeying for DWDM transmission," in ECOC'03, Th.2.6.5, 2003.

[2] O. Vassilieva, et al., "Non-linear tolerant and spectrally efficient $86 \mathrm{Gbit} / \mathrm{s}$ RZ-DQPSK format for a system upgrade," in $O F C^{\prime} 03$, ThE7, 2003

[3] M. Daikoku, et al., "100 Gbit/s DQPSK transmission experiment without OTDM for 100G Ethernet transport," in ECOC'06, PDP36, 2006.

[4] T. Tokle, et al., "Investigation of multilevel phase and amplitude modulation formats in combination with polarisation multiplexing up to 240 Gbit/s," IEEE Photon. Technol. Lett., vol. 18, no. 20 , pp. $2090-2092,2006$

[5] T. Tokle, et al., "160 Gbit/s single channel data generation using multilevel optical RZ-DPSK-ASK modulation and optical time division multiplexing," in ECOC'05, We3.2.5, 2005.

[6] K. Sekine, et al., "40 Gbit/s, 16-ary (4 bit/symbol) optical modulation/demodulation scheme," Electron. Lett., vol. 41, no. 7, pp. 430-432, 2005

[7] M. Serbay, et al., " 42.8 Gbit/s 16-ary Inverse-RZ-QASK-DQPSK transmission experiment operating at 10.7 Gbaud without polmux," in $O F C^{\prime} 07$, OThL2, 2007.

[8] H. Weber, et al., "Single channel $1.28 \mathrm{Tbit} / \mathrm{s}$ and $2.56 \mathrm{Tbit} / \mathrm{s}$ DQPSK transmission," Electron. Lett., vol. 42, no. 3, pp. 67-68, 2006. 